\title{
A case of small-cell esophageal cancer with chronic renal failure undergoing hemodialysis safely treated with cisplatin and etoposide
}

\author{
Kentaro Murakami • Yasunori Akutsu • Yukimasa Miyazawa • \\ Kiyohiko Shuto · Toru Shiratori • Masaya Uesato • \\ Isamu Hoshino · Taito Aoki · Hisahiro Matsubara
}

Received: 8 April 2011/Accepted: 29 June 2011/Published online: 18 July 2011

(C) The Author(s) 2011. This article is published with open access at Springerlink.com

\begin{abstract}
A 54-year-old male undergoing hemodialysis was admitted to our hospital because of difficulty in swallowing. Esophagography and esophageal endoscopy revealed an irregular ulcerated lesion in the cervical esophagus. It was diagnosed as a small-cell esophageal cancer from the biopsy sample. Computed tomography showed a tumor infiltrating the trachea and a few lymph node metastases in the cervix, upper mediastinum, and abdomen. The patient was started on chemotherapy with cisplatin (CDDP) and etoposide (VP-16), which had been reported to be effective for small-cell lung cancer. The patient was treated with CDDP $\left(80 \mathrm{mg} / \mathrm{m}^{2}\right)$ on day 1 and VP-16 $\left(100 \mathrm{mg} / \mathrm{m}^{2}\right)$ on days 1,3 , and 5 , every 4 weeks. On the days of chemotherapy, hemodialysis was started as soon as possible after completion of administration of the agents. No severe side effects were observed. After 4 courses of therapy, the patient achieved a partial response.
\end{abstract}

Keywords Small-cell cancer - Esophagus ·

Hemodialysis · Cisplatin · Etoposide

K. Murakami ( $\square) \cdot$ Y. Akutsu · Y. Miyazawa · K. Shuto ·

T. Shiratori - M. Uesato - I. Hoshino - H. Matsubara

Department of Frontier Surgery, Graduate School of Medicine,

Chiba University, 1-8-1 Inohana, Chuo-ku,

Chiba 260-8670, Japan

e-mail: murakami2621@gmail.com

Y. Akutsu

e-mail: yakutsu@faculty.chiba-u.jp

T. Aoki

Makuhari Gastrointestinal Clinic, Chiba, Japan

\section{Introduction}

Small-cell esophageal cancer (SCEC) is a rare tumor accounting for approximately $1 \%$ of all esophageal malignancies [1]. SCEC behaves aggressively with early systemic metastasis, so prognosis is poor. Although combined treatment including surgery, chemotherapy, and radiotherapy has been attempted, no optimum treatment approach for SCEC has been established. Recently, it has been regarded as a systemic disease, and systemic chemotherapy has become the treatment of choice. Because of their histopathological similarity, the chemotherapy used for small-cell lung cancer (SCLC) is often chosen for SCEC.

Because pharmacokinetics in patients undergoing hemodialysis differ from those in patients with normal renal function, in hemodialysis patients it is difficult to determine a safe and effective dose and dosing schedule for anticancer agents, including the optimum timing of hemodialysis. Therefore an appropriate regimen of chemotherapy for patients undergoing hemodialysis has not been clearly established.

There are no reports in the literature of chemotherapy for patients with SCEC maintained on hemodialysis. We herein report a case with SCEC on hemodialysis to whom cisplatin (CDDP) combined with etoposide (VP-16) was administered safely and effectively.

\section{Case report}

Because of chronic glomerulonephritis a 54-year-old male had been maintained on hemodialysis for approximately 10 years at his local hospital. He presented with difficulty in swallowing and was examined by endoscopy in November 
2007, which led to diagnosis of esophageal cancer. Biopsy specimens revealed the lesions to be SCEC (Fig. 1c). He was admitted to our hospital in December, and esophagography showed an elevated mass with ulceration, measuring $7 \mathrm{~cm}$ in diameter, in the cervical esophagus (Fig. 1a). Esophageal endoscopy revealed an irregularly elevated lesion occupying approximately two-thirds of the circumference, with ulceration, $18-23 \mathrm{~cm}$ from his incisors (Fig. 1b). Computed tomography (CT) revealed a solid mass, measuring $28 \mathrm{~mm}$ in a diameter, in the cervical esophagus, which invaded the trachea, and lymph node enlargement in the cervical paraesophageal area, the supraclavicular area, the tracheobronchial area, and the area along the lesser curvature (Fig. 1d). Blood chemistry analysis revealed an elevated serum level $(107.9 \mathrm{pg} / \mathrm{ml})$ of pro-gastrin-releasing peptide (Pro-GRP).

The lesion was diagnosed as T4, N1, M1, and stage IV (UICC TNM classification). Because the tumor was judged to be unresectable, chemotherapy was selected as the treatment for this case. We knew CDDP and VP-16 were effective in the treatment of SCLC, leading us to a decision to use these anticancer agents for the systemic chemotherapy. The chemotherapeutic regimen consisted of CDDP on day 1 and VP-16 on days 1, 3 and 5, every 4 weeks. In the conventional schedule of CDDP and VP-16 therapy for SCLC, VP-16 is administered on days 1-3. However, we wanted to match the day of administration with the day of dialysis without reducing the total dosage of VP-16. Therefore, we chose a different schedule of
VP-16, and administered it on days 1, 3, and 5. The administration schedule for the two agents on day 1 consisted of intravenous injection of VP-16 in $500 \mathrm{ml}$ normal saline for $120 \mathrm{~min}$ and of CDDP for $60 \mathrm{~min}$ after the end of VP-16 infusion, and no hydration was performed. For this patient, standard-dose chemotherapy was administered, consisting of CDDP and VP-16 at 80 and $100 \mathrm{mg} / \mathrm{m}^{2}$, respectively. He was hemodialyzed with a cellulose triacetate dialyzer three times per week, for approximately $4 \mathrm{~h}$ per hemodialysis session. On the days of chemotherapy, hemodialysis was started as soon as possible (within $30 \mathrm{~min}$ ) after completion of administration of the chemotherapeutic agents (Fig. 2) [2].

\section{Effects of chemotherapy}

After the fourth course was finished, the lesion was evaluated. A partial response was noted, and the patient was able to eat solid food after the treatment. Esophagography showed that the tumor was reduced to $4 \mathrm{~cm}$ and had become flatter (Fig. 3a). Esophageal endoscopy revealed that the tumor which projected into the lumen had almost disappeared. Only a mildly irregular mucosa and a minute elevated mass remained in the cervical esophagus, 22-24 cm from his incisors (Fig. 3b). A CT scan showed a solid mass which had decreased to $21 \mathrm{~mm}$ in size, in the cervical esophagus, which did not seem to invade the trachea. The size of the lymph node swelling had also decreased (Fig. 3c). The serum level of Pro-GRP fell after
Fig. 1 a Esophagography revealed an elevated mass with ulceration, measuring $7 \mathrm{~cm}$ in diameter, in the cervical esophagus. b Esophageal endoscopy revealed an irregularly elevated lesion occupying approximately twothirds of the circumference, with ulceration, $18-23 \mathrm{~cm}$ from his incisors. c Histopathologic examination of the biopsy specimens revealed that the tumor was composed of small, round-to-oval cells with hyperchromatic nuclei and scant cytoplasm $(\mathrm{H} \& \mathrm{E}$ stain $\times 100)$. d Computed tomography (CT) revealed a solid mass,

measuring $28 \mathrm{~mm}$ in a diameter, in the cervical esophagus, which invaded the trachea
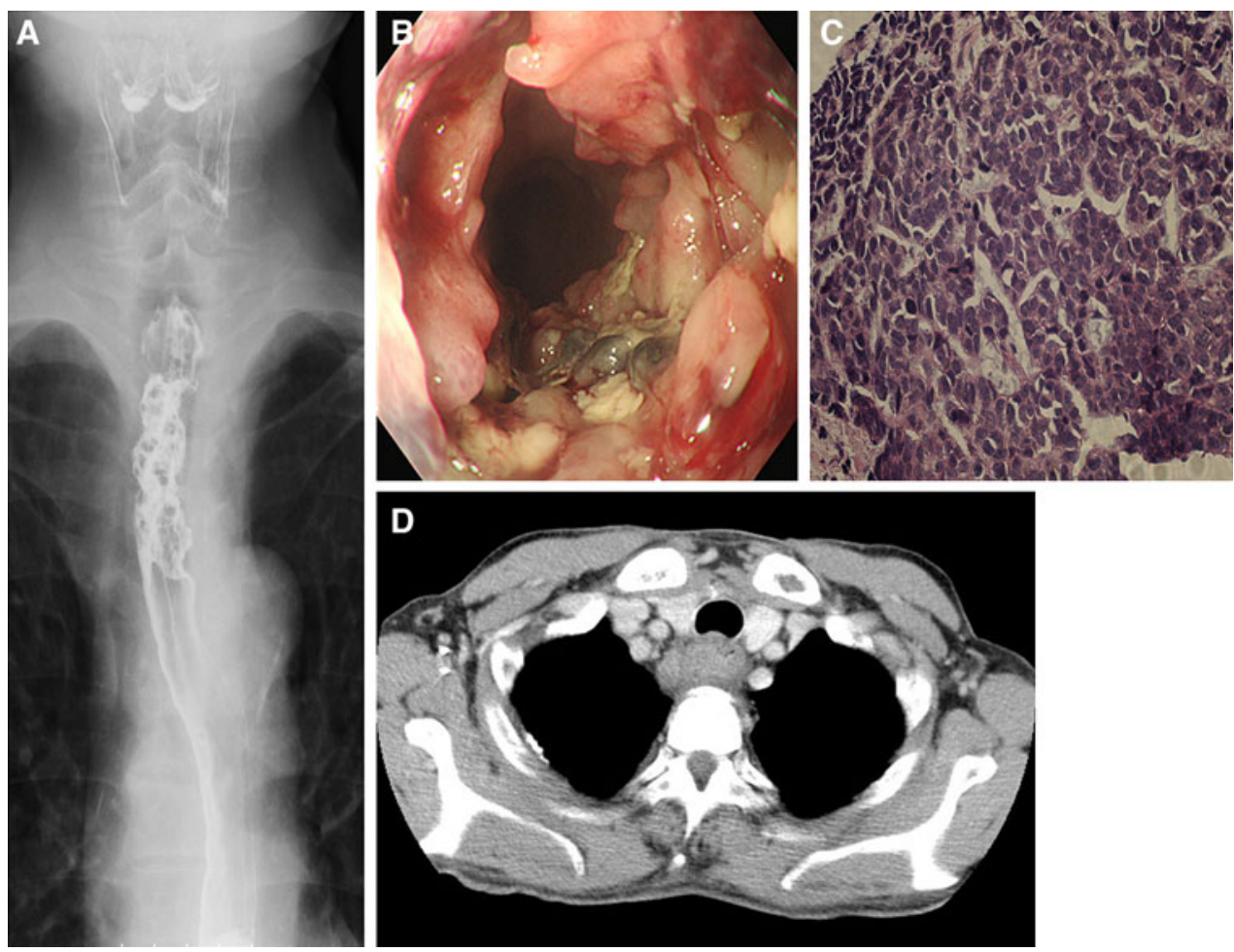

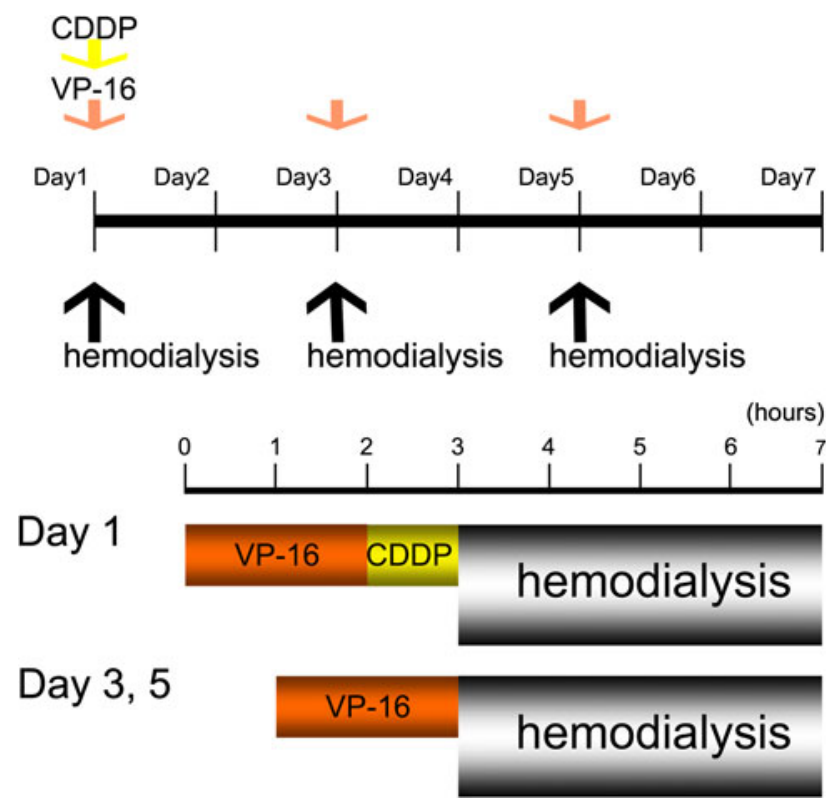

Fig. 2 The chemotherapeutic regimen consisted of CDDP on day 1 and VP-16 on days 1,3 , and 5 , every 4 weeks. The schedule of administration of the two agents on day 1 consisted of intravenous injection of VP-16 for $120 \mathrm{~min}$ then CDDP for $60 \mathrm{~min}$ after the end of VP-16 infusion. This patient was hemodialyzed three times per week, for approximately $4 \mathrm{~h}$ per hemodialysis session. On the days of chemotherapy, hemodialysis was started as soon as possible (within $30 \mathrm{~min}$ ) after completion of administration of the agents

the course of chemotherapy and rose after the interval between chemotherapy. The minimum value of pro-GRP was $38.5 \mathrm{pg} / \mathrm{ml}$, but after 4 courses of this regimen, the serum level of Pro-GRP was $165.7 \mathrm{pg} / \mathrm{ml}$. An additional 5 courses of treatment were administered. At that time, examinations had shown no progressive growth of the tumor. However, 12 months after the initial treatment disease progression was observed. The CT scans showed regrowth of the main tumor and multiple cervical lymph node metastases. Because the patient's pain had worsened because of enlargement of the lymph node metastases, and his performance status had abruptly worsened, palliative radiation (30 Gy) was administered for the purpose of pain relief. However, because the patient could not eat and was markedly emaciated, we judged that the continuation of the treatment would be difficult, so best supportive care was provided. One month after stopping treatment, his body weight continued to decrease, and marked general edema occurred. The patient died as a result of disease progression.

\section{Toxicity}

With regard to the toxicity of this chemotherapy regimen, the patient complained of only mild nausea and loss of appetite (Grade 1 by NCI-CTCAE v4.0). Although occasional afebrile neutrocytopenia (Grade 3) was apparent from the blood chemistry data, we were easily able to treat it with granulocyte colony stimulating factor (G-CSF). The patient had nephrogenic anemia (hemoglobin $7.2 \mathrm{~g} / \mathrm{dl}$, packed cell volume $23.0 \%$ ) just before chemotherapy, and so experienced exacerbation of his anemia (Grade 4) as a result of the chemotherapy. Therefore, a blood transfusion was performed twice during the course of treatment. These complications were tolerable.

\section{Pharmacokinetics}

We measured plasma levels of total CDDP (t-CDDP) and VP-16. Venous blood samples were collected into tubes just before and after drug administration, then $0.5,1,2,3$, 4, 5, $12 \mathrm{~h}, 1$ (24 h), 2, 3, 4 days, and 1, 2, 3 weeks after administration. After separating plasma from each sample, the concentrations of t-CDDP and VP-16 were measured in a portion of the plasma. As shown in Figs. 4 and 5, we compared the time-concentration curves of t-CDDP and VP-16 between the patient with renal insufficiency and those with normal renal function. Figures $4 b$ and $5 b$ were provided by Dr Masumi Sawada and Dr Akira Wakui, respectively. The pharmacokinetic parameters, including half-life $\left(t_{1 / 2}\right)$ and area under the curve (AUC), were calculated for CDDP and VP-16.

The t-CDDP concentration decreased abruptly immediately after administration of CDDP ( $\alpha$ phase); afterward it decreased more slowly ( $\beta$ phase) (Fig. 4a). This biphasic pattern of the pharmacokinetics was similar to that for patients with normal renal function (in a phase 1 clinical trial) [3] (Fig. 4b). The t-CDDP concentration on initiation of hemodialysis was $2.31 \mu \mathrm{g} / \mathrm{ml}$. The maximum drug concentration $\left(C_{\max }\right)$ (CDDP $50 \mathrm{mg} /$ body) as shown in Fig. $4 \mathrm{~b}$ was higher than that observed in our data (CDDP $120 \mathrm{mg} /$ body). However, in other studies, it was reported tha the $C_{\max }$ was $1.40,0.98,1.07 \mu \mathrm{g} / \mathrm{ml}$ (CDDP $80 \mathrm{mg} / \mathrm{body}$ ) [4], $3.31 \mu \mathrm{g} / \mathrm{ml}\left(\mathrm{CDDP} 80 \mathrm{mg} / \mathrm{m}^{2}\right)$ [5], and $2.90 \mu \mathrm{g} / \mathrm{ml}$ (CDDP $100 \mathrm{mg} / \mathrm{m}^{2}$ ) [6]. The reason why the value of $C_{\max }$ in a phase 1 clinical trial tends to be high is unclear. However, our data were consistent with those in previous reports. It decreased to $1.39 \mu \mathrm{g} / \mathrm{ml}$ at $0.5 \mathrm{~h}$, and was $1.56 \mu \mathrm{g} / \mathrm{ml} 4 \mathrm{~h}$ after the initiation. Most t-CDDP was removed in the first $30 \mathrm{~min}$ of dialysis. The lower limit of detection of $\mathrm{t}$-CDDP was $0.1 \mu \mathrm{g} / \mathrm{ml}$. The maximum $\mathrm{t}$-CDDP concentration was $2.31 \mu \mathrm{g} / \mathrm{ml}$, and it had decreased to $0.17 \mu \mathrm{g} / \mathrm{ml} 28$ days after the administration of CDDP. CDDP remained in the plasma for at least 28 days, but the last concentration approached the lower limit of detection. We also measured the plasma levels of t-CDDP in the first and second courses and compared each point in both courses. The t-CDDP concentrations in the second course were significantly higher than those in the first 
Fig. 3 a Esophagography showed the tumor was reduced to $4 \mathrm{~cm}$ and had become flatter. b Esophageal endoscopy revealed that the tumor that projected into the lumen had almost disappeared. Only a mildly irregular mucosa and a minute elevated mass remained in the cervical esophagus, 22-24 cm from his incisors. c A CT scan showed a solid mass which had decreased to $21 \mathrm{~mm}$ in size, in the cervical esophagus, which did not seem to invade the trachea. The size of lymph node swelling had also decreased
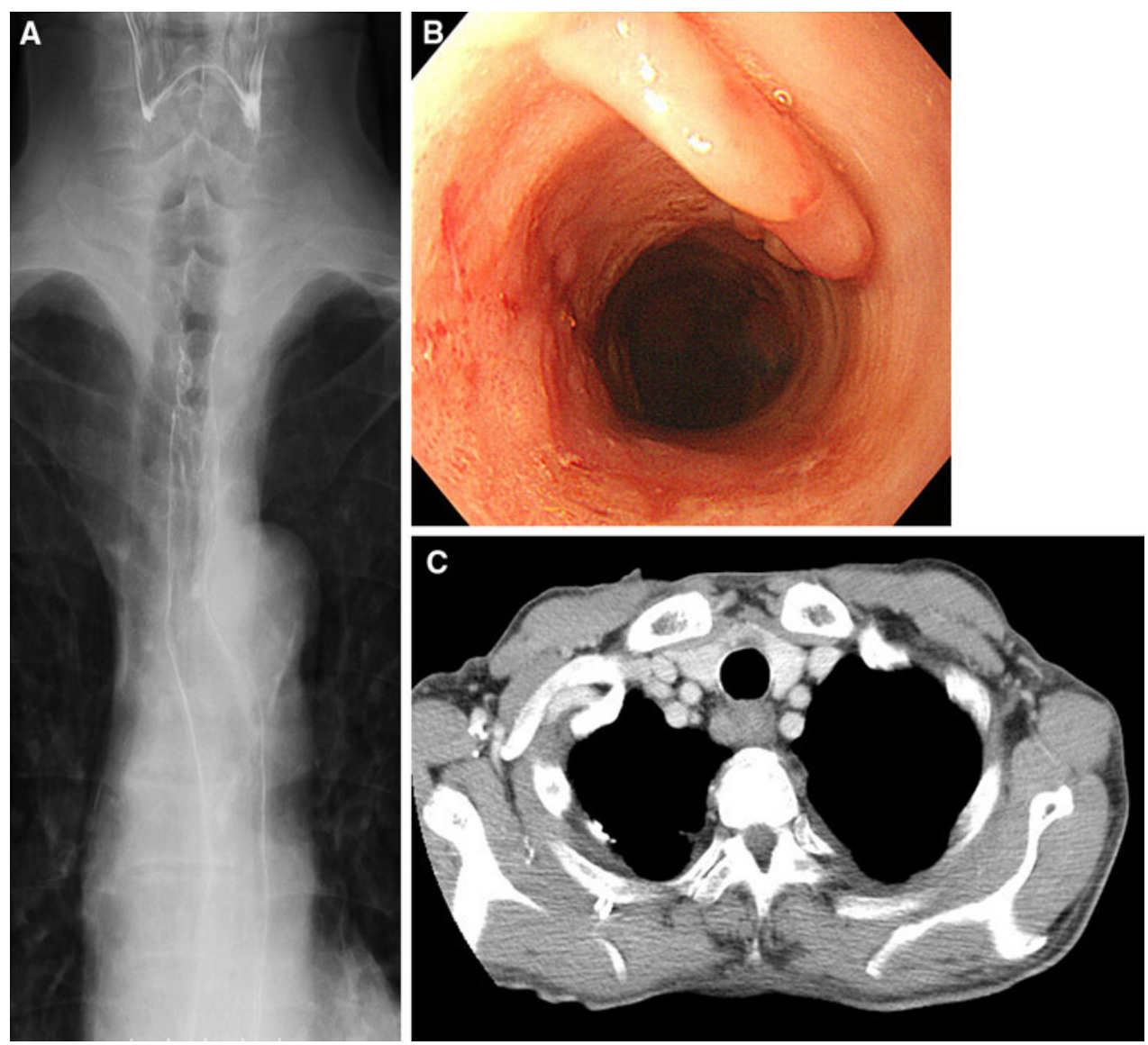

course (Wilcoxon $t$ test, $P<0.01$ ), indicating a cumulative effect of CDDP in this regimen. The $t_{1 / 2 \alpha}$ was $0.036 \mathrm{~h}$, and $t_{1 / 2 \beta}$ was $119 \mathrm{~h}$ for the first course of CDDP. In contrast, the $t_{1 / 2 \alpha}$ was $0.411 \mathrm{~h}$ and $t_{1 / 2 \beta}$ was $164 \mathrm{~h}$ for the second course. The AUC was 265 and $363 \mu \mathrm{g} \mathrm{h} / \mathrm{ml}$ in the first and second courses, respectively. The $t_{1 / 2}$ was extended and the AUC was increased in the second course in comparison with the first course. Many reports have described that the $t_{1 / 2 \beta}$ was approximately $100 \mathrm{~h}$ in patients with normal renal function [6], and Tokunaga et al. [4] reported that the $t_{1 / 2 \alpha}$ for CDDP was $0.53 \pm 0.07 \mathrm{~h}, t_{1 / 2 \beta}$ was $94.2 \pm 11.6 \mathrm{~h}$, and AUC was $71.3 \pm 6.26 \mu \mathrm{g} \mathrm{h} / \mathrm{ml}$ after administration of $90.8 \pm 6.34 \mathrm{mg}$ to patients with normal renal function. In this case, we obtained a similar $t_{1 / 2}$ value, but the AUC tended to be greater than for patients with normal renal function.

The VP-16 maximum concentrations were 9.96, 10.44, and $10.45 \mu \mathrm{g} / \mathrm{ml}$ after the three administrations and rapidly decreased to $5.06,6.07$, and $5.76 \mu \mathrm{g} / \mathrm{ml} 4 \mathrm{~h}$ after administration ( $\alpha$ phase). The concentrations then decreased gradually ( $\beta$ phase) (Fig. 5a). The pharmacokinetics had a biphasic pattern which was also comparable with the curves for patients with normal renal function (in a phase 1 clinical trial) [7] (Fig. 5b). The concentration had decreased to
$1.45 \mu \mathrm{g} / \mathrm{ml}$ at $24 \mathrm{~h}$ and $0.62 \mu \mathrm{g} / \mathrm{ml}$ at $48 \mathrm{~h}$ after administration, which was approaching the lower limit of detection. Almost all of the VP-16 in the plasma was excreted within $48 \mathrm{~h}$. Comparison of each point after the first and second administrations revealed the VP-16 concentrations were not significantly different (Wilcoxon $t$ test, $P=0.068$ ), and no significant cumulative effect caused by the alternate-day administration for 3 days was apparent. We also evaluated the pharmacokinetic parameters of VP-16 in this patient. The $t_{1 / 2 \alpha}$ was $2.31 \mathrm{~h}$ and $t_{1 / 2 \beta}$ was $17.2 \mathrm{~h}$ after the first administration in the first course of treatment, and the $t_{1 / 2 \alpha}$ was $3.10 \mathrm{~h}$ and $t_{1 / 2 \beta}$ was $16.9 \mathrm{~h}$ after the second administration during the first course. The AUC values were 128 and $139 \mu \mathrm{g} \mathrm{h} / \mathrm{ml}$, respectively, after the first and second administrations during the first course. There was little difference between $t_{1 / 2}$ and AUC after the first and second administrations. On the other hand, Wakui et al. [7] reported that the $t_{1 / 2 \alpha}$ was 0.13 and $0.30 \mathrm{~h}, t_{1 / 2} \beta$ was 4.85 and $4.01 \mathrm{~h}$, and the AUC was 95.7 and $94.8 \mu \mathrm{g} \mathrm{h} / \mathrm{ml}$, respectively, after the administration of $120 \mathrm{mg} / \mathrm{m}^{2}$ in patients with normal renal function (based on a phase 1 clinical trial). This suggested that there was a tendency for both the $t_{1 / 2}$ and AUC to increase in this dialysis patient in comparison with patients with normal renal function. 


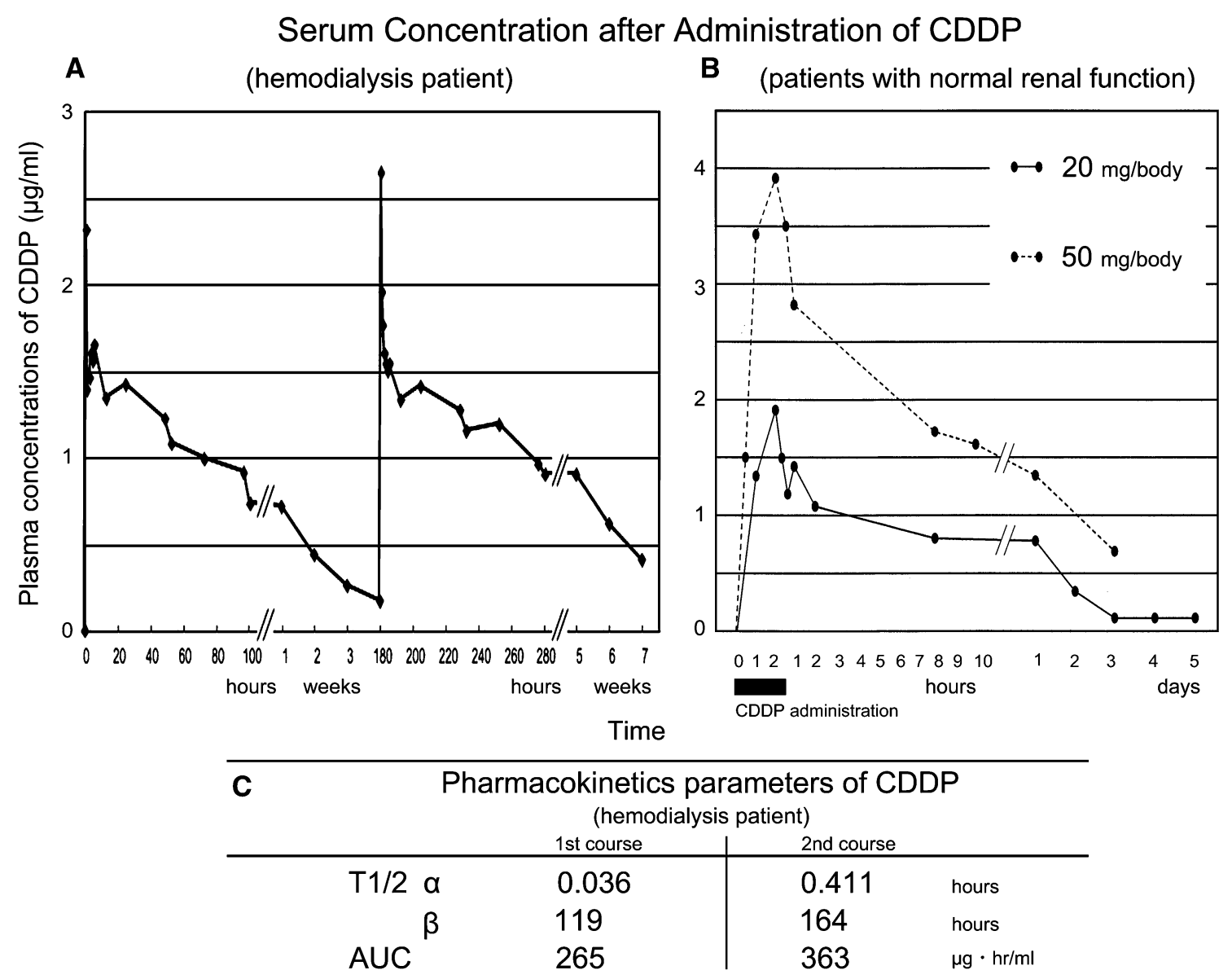

Fig. 4 The t-CDDP concentration decreased abruptly immediately after administration of CDDP ( $\alpha$ phase) and then decreased slowly ( $\beta$ phase). This biphasic pattern of the pharmacokinetics was similar to that for patients with normal renal function. a The hemodialysis

patient. b Patients with normal renal function (the figure was provided by Dr Masumi Sawada, Osaka University). c Pharmacokinetic parameters for CDDP

\section{Discussion}

SCEC is a rare tumor accounting for approximately $1 \%$ of all esophageal malignancies [1]. There is no established treatment for the disease, but combination chemotherapy consisting of CDDP and VP-16, such as is given for SCLC, is generally administered.

It is well known that patients with chronic renal failure suffer from malignancies at high frequency, as reported by Matas et al. [8]. With the increase in the number of dialysis patients, cases in which malignancies are detected during a dialysis period have increased.

When an anticancer agent is administered to a patient with chronic renal failure, it is necessary to consider the metabolic pathway of the anticancer agent and to adjust the administration method in order to reduce side effects while maintaining antitumor efficacy. CDDP is a chemotherapeutic agent that is excreted into urine. Its half-life $\left(t_{1 / 2}\right)$ isapproximately $100 \mathrm{~h}$, which is longer than that of other anticancer agents, so we were concerned about accumulation of the drug in the patient with chronic renal failure. Some papers have indicated that the dose should be reduced to $50 \%$ in patients with renal insufficiency $[9,10]$. The major route of excretion of VP-16 is the bile and stool. Urinary excretion by patients with normal renal function is $30-40 \%$ after intravenous administration. The dose that should be given to patients with chronic renal failure is controversial, and some papers have reported a need to reduce the dose [9, 10]. However, it is controversial whether antitumor activity is retained after dose reduction. There have been of reports on CDDP and VP-16 chemotherapy for a dialysis patients with SCEC.

In this case, we first discussed the safety of this regimen. The patient complained of mild nausea and appetite loss, but his dietary intake was reduced by half for approximately 3 days only after administration of CDDP. Although frequent anemia and neutrocytopenia were observed, the toxicity was tolerable. The nadir was shown to be 16-23 days after administration, and did not last longer than in patients with normal renal function. We 
Serum Concentration after Administration of VP-16

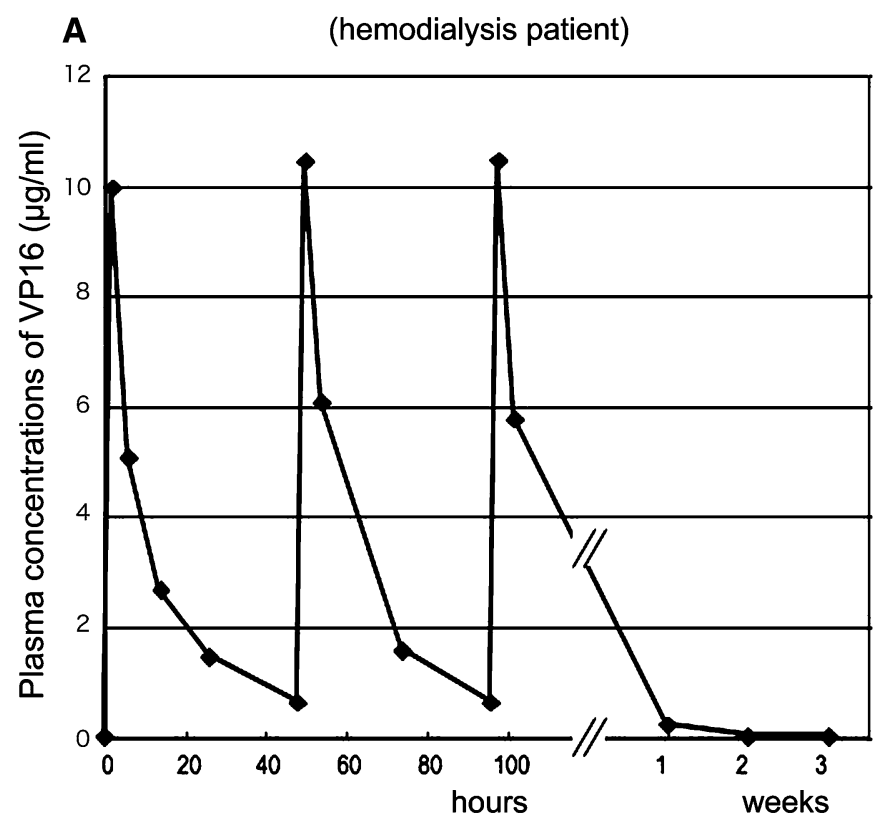

B (patients with normal renal function)

Time

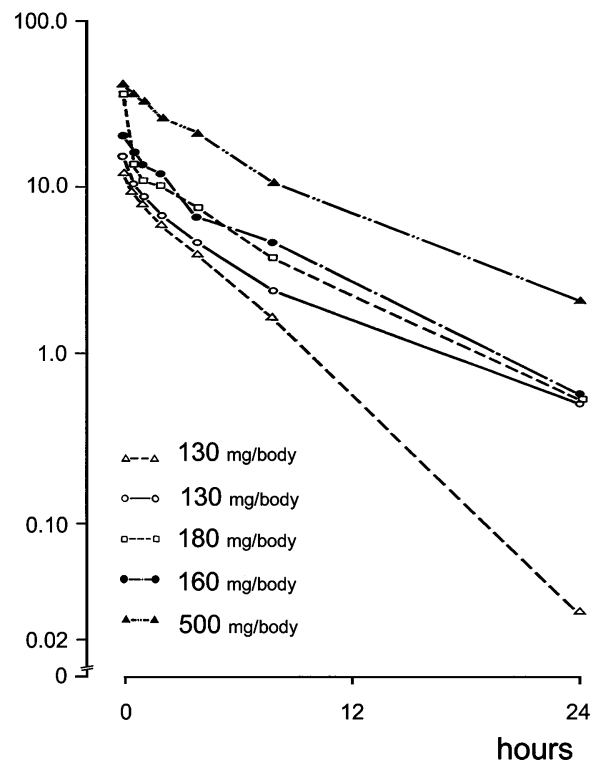

\begin{tabular}{lll|ll}
\hline C & \multicolumn{5}{c}{$\begin{array}{c}\text { Pharmacokinetics parameters of VP-16 } \\
\text { (hemodialysis patient) } \\
\text { 1st course }\end{array}$} & 2nd course & \\
& & 2.31 & 3.10 & hours \\
T1/2 & $\alpha$ & 17.2 & 16.9 & hours \\
& $\beta$ & 128 & 139 & $\mu \mathrm{g} \cdot \mathrm{hr} / \mathrm{ml}$
\end{tabular}

Fig. 5 The VP-16 concentration decreased rapidly during the first $4 \mathrm{~h}$ after administration ( $\alpha$ phase) and then decreased gradually ( $\beta$ phase). The biphasic pattern of the pharmacokinetics was comparable with that for patients with normal renal function. a The hemodialysis

suggest that this regimen can be safely administered even to dialysis patients.

We also studied the efficacy of the regimen in this case. SCEC is classified as limited disease (LD) or extensive disease (ED) according to the Veterans Administration Lung Group staging system [11]. It has been reported that the median survival time (MST) for ED is 7.0 months, and that 1-year survival is $29.3 \%$ [12]. In our case the patient was staged as ED, and he maintained stable disease (SD) for 12 months, and survived for 13 months. In comparison with the previous report, the curative effect in this case was slightly better. Our findings therefore suggest that this regimen is effective for treatment of dialysis patients.

We also studied the good results in terms of safety and efficacy and their relationship to the pharmacokinetics. The timing of hemodialysis initiation after administration of the anticancer agent and the doses of the anticancer agents were very important in terms of the safety and efficacy of the treatment. patient. b Patients with normal renal function (the figure was provided by Dr Akira Wakui, Tohoku University). c Pharmacokinetics parameters for VP-16

In terms of safety, it is necessary to prevent serious side effects associated with CDDP accumulation. The freeCDDP (f-CDDP) could be removed easily by hemodialysis. However, protein binding of CDDP in the plasma is approximately $90 \%$, and f-CDDP binds to plasma proteins immediately and strongly [13]. Protein-bound CDDP is difficult to remove effectively. In this case, the f-CDDP was removed quickly by hemodialysis immediately after its administration, thus blocking some of the binding to the plasma proteins. As a result, the t-CDDP concentrationtime curve and the value of $t_{1 / 2}$ in this case were almost equal to those for patients with normal renal function reported previously. On the basis of a literature review, the AUC for CDDP was higher than for patients with normal renal function. It is said that the AUC of CDDP correlates with its side effects. However, there are individual differences in clinical side effects. In this case, there were no severe side effects. However, it should be kept in mind that there is a risk of severe side effects, especially for patients 
with compromised renal function. Approximately 4 weeks were required for CDDP to be excreted completely. The dosing interval in this regimen was 4 weeks, but the cumulative effect of CDDP was clear upon comparison of the pharmacokinetics in the first and second courses. In this case, there was no clinically significant increase in the side effects during the second course, but it is necessary to take care when administering this regimen on this schedule. It has been reported that the efficacy of platinum-containing drugs depends on both the length of exposure and the concentration, and the anti-tumor effect was likely to correlate with the AUC, because it contains both elements. When CDDP is administered to a dialysis patient, the AUC depends not only on the dose of drug administered but also on the timing of dialysis initiation. In this case, the AUCs were increased compared with patients with normal renal function. This may be one of the reasons we obtained a slightly better anti-tumor effect.

We also analyzed the pharmacokinetics of VP-16, which is excreted mainly through the bile and stool, only slightly in the urine, unlike CDDP. Because a small percentage of the administered VP-16 is excreted into the urine, the dose of the anticancer agent and the dialysis schedule may not affect the pharmacokinetics significantly. However, some papers recommended dose reduction of VP-16 for dialysis patients, because of its poor dialyzability. In this case, the maximum concentration was almost equal to that in patients with normal renal function, but the $t_{1 / 2}$ was longer. A tendency for VP-16 to accumulate in the hemodialysis patients was noted. However, a large quantity was excreted in $<48 \mathrm{~h}$, and it was revealed that there was no cumulative effect by comparison of the pharmacokinetics after each administration. In terms of safety, the usual dose could be safely administered by starting hemodialysis soon after administration of the drug. In terms of efficacy, the AUC was larger than that for patients with a normal renal function, and thus there is a possibility that we obtained a slightly better anti-tumor effect compared to patients with normal renal function.

Chemotherapy using this regimen was performed safely without severe side effects, and almost equal anti-tumor efficacy was observed compared with patients with normal renal function. On the basis of the above-mentioned pharmacokinetics, it was suggested that the good results were because of the time-concentration relationship, which resembled that for patients with normal renal function. In terms of safety and efficacy, this regimen, which consisted of the usual dose of chemotherapeutic agents, and the initiation of dialysis immediately after administration of the agents, was considered to be reasonable.

In conclusion, this full-dose combination chemotherapy regimen comprising CDDP and VP-16 seems to be safe and effective against esophageal SCEC in patients undergoing regular hemodialysis. Further study is needed for selection of suitable chemotherapeutic regimens, the optimum dose of each drug, and the timing of hemodialysis.

Acknowledgments The authors thank Dr Masumi Sawada and Dr Akira Wakui for providing the figures (Figs. 4b and 5b) from their phase 1 clinical trials, and Nippon Kayaku Co., Ltd., Tokyo, Japan, for help in performing the pharmacokinetic analysis.

Open Access This article is distributed under the terms of the Creative Commons Attribution Noncommercial License which permits any noncommercial use, distribution, and reproduction in any medium, provided the original author(s) and source are credited.

\section{References}

1. The Japanese Society for Esophageal Disease. Comprehensive registry of esophageal cancer in Japan $(1998,1999)$ and longterm results of esophagectomy in Japan (1988-1997). 3rd ed.

2. Watanabe R, Takiguchi Y, Moriya T, Oda S, Kurosu K, Tanabe N, Tatsumi K, Nagao K, Kuriyama T. Feasibility of combination chemotherapy with cisplatin and etoposide for haemodialysis patients with lung cancer. Br J Cancer. 2003;88:25-30.

3. Sawada M, Okudaira M, Matsui Y, Nishiura H, Yanagida T. About the pharmacokinetics of cisplatin. Obstet Gynecol Pract. 1983;32:2117-23.

4. Tokunaga J, Kikukawa H, Nishi K, Kitani K, Fujii J, Honda J, Wada Y, Ueda S, Nakano M. Pharmacokinetics of cisplatin and methotrexate in a patient suffering from advanced ureteral tumor accompanied by chronic renal failure, undergoing combined hemodialysis and systemic M-VAC chemotherapy. Gan To Kagaku Ryoho. 2000;27:2079-85.

5. Ikeda K, Terashima M, Kawamura H, Takiyama I, Koeda K, Takagane A, Sato N, Ishida K, Iwaya T, Maesawa C, Yoshinari $\mathrm{H}$, Saito K. Pharmacokinetics of cisplatin in combined cisplatin and 5-fluorouracil therapy: a comparative study of three different schedules of cisplatin administration. Jpn J Clin Oncol. 1998;28: $168-75$.

6. Johnsson A, Höglund P, Grubb A, Cavallin-Ståhl E. Cisplatin pharmacokinetics and pharmacodynamics in patients with squamous-cell carcinoma of the head/neck or esophagus. Cancer Chemother Pharmacol. 1996;39:25-33.

7. Wakui A, Yokoyama M, Takahashi H, Yoshida Y, Sakata Y, Sato S, Kano A, Kawamoto K, Hashimoto S, Konno K, et al. A phase I study of VP-16-213 (VP, etoposide) by single and 5-day intravenous administration. Gan To Kagaku Ryoho. 1986;13:319-29.

8. Matas AJ, Simmons RL, Kjellstrand CM, Buselmeier TJ, Najarian JS. Increased incidence of malignancy during chronic renal failure. Lancet. 1975;19:883-6.

9. Tomita M, Aoki Y, Tanaka K. Effect of haemodialysis on the pharmacokinetics of antineoplastic drugs. Clin Pharmacokinet. 2004;43:515-27.

10. Janus N, Thariat J, Boulanger H, Deray G, Launay-Vacher V. Proposal for dosage adjustment and timing of chemotherapy in hemodialyzed patients. Ann Oncol. 2010;21:1395-403.

11. Zelen M. Keynote address on biostatistics and data retrieval. Cancer Chemother Rep. 1973;4:31-42.

12. Lv J, Liang J, Wang J, Wang L, He J, Xiao Z, Yin W. Primary small cell carcinoma of the esophagus. J Thorac Oncol. 2008;3: $1460-5$.

13. Aronoff GR. Drug prescribing in renal failure. 5th ed. 2007. p. 98. 